\title{
Effect of Packaging on Microbial Survival and Physicochemical Characteristics of Non-Thermally Preserved Green Spanish-Style Olives ${ }^{+}$
}

\author{
Aikaterini Tzamourani ${ }^{1}$, George Economou-Petrovits ${ }^{2}$, Stavros Panagiotidis ${ }^{2}$, George-John Nychas ${ }^{1}$ and \\ Efstathios Z. Panagou 1,*
}

Citation: Tzamourani, A.; Economou-Petrovits, G.; Panagiotidis, S.; Nychas, G.-J.; Panagou, E.Z. Effect of Packaging on Microbial Survival and Physicochemical Characteristics of Non-Thermally Preserved Green Spanish-Style Olives. Proceedings 2021, 70, 61. https://doi.org/10.3390/ foods_2020-07594

Published: 9 November 2020

Publisher's Note: MDPI stays neutral with regard to jurisdictional claims in published maps and institutional affiliations.

\section{Copyright: $₫ 2020$ by the authors.}

Licensee MDPI, Basel, Switzerland. This article is an open access article distributed under the terms and conditions of the Creative Commons Attribution (CC BY) license (http://creativecommons.org/licenses /by/4.0/).
1 Laboratory of Microbiology and Biotechnology of Foods, Department of Food Science and Human Nutrition, School of Food and Nutritional Sciences, Agricultural University of Athens, Iera Odos 75, 11855 Athens, Greece; tzam.katerina@gmail.com (A.T.); george.nychas@gmail.com (G.-J.N.)

2 PELOPAC S.A., Block 38, NB1A Street, Thessaloniki Industrial Area, 57022 Sindos, Greece; economou@pelopac.gr (G.E.-P.); spanagiotidis@pelopac.gr (S.P.)

* Correspondence: stathispanagou@aua.gr; Tel.: +30-210-5294693

† Presented at the 1st International Electronic Conference on Food Science and Functional Foods, 10-25 November 2020. Available online: https://foods_2020.sciforum.net/.

\begin{abstract}
Spanish-style green olives are among the main trade preparations in the international market. The purpose of this work was to investigate the effect of the modified atmosphere packaging of Spanish-style green olives in multi-layered pouches on the microbiological and physicochemical characteristics of olives. Green pitted olives of cv. Conservolea and Halkidiki were packaged in high-barrier multi-layered pouches under modified atmospheres and stored at room temperature for 12 months. Microbiological (lactic acid bacteria (LAB), yeasts, and Enterobacteriaceae) and physicochemical changes ( $\mathrm{pH}$, acidity, salt content, texture and color) were monitored throughout storage together with sensory assessment. Results showed that the initial microbiota consisted of LAB (5.9-6.3 log CFU/g) and yeasts (4.8-5.3 log CFU/g). Yeasts started to decline and could not be detected after 90 days of storage. In the end, LAB dominated in populations exceeding 5.4 and $6.1 \log$ CFU/g for cv. Halkidiki and Conservolea, respectively. No Enterobacteriaceae could be detected in both olive varieties. In addition, $\mathrm{pH}$ values increased gradually from 3.51 to 4.19 , the mean acidity was ca. $0.2 \%$ (expressed as lactic acid) and the salt content fluctuated between 2.0 $4.4 \%$. Color parameters $L^{*}$ (luminance), $a^{*}$ (greenness/redness) and $b^{*}$ (yellowness/blueness) did not change during storage in both varieties. Texture analysis showed a decrease in olive hardness from $15-20 \mathrm{~N}$ to $8 \mathrm{~N}$ throughout storage. Sensory analysis revealed that $\mathrm{cv}$. Halkidiki olives were crisper with increased fibrousness compared to cv. Conservolea.
\end{abstract}

Keywords: green olives; modified atmosphere packaging; multi-layered pouches; lactic acid bacteria; yeasts

\section{Introduction}

Table olives are one of the oldest and most popular fermented foods with significant economic significance in Mediterranean countries [1]. Nowadays, there is an increasing trend to use plastic packaging due to reduced weight, lower costs, flexibility, and convenience, while modified atmosphere packaging (MAP) has been used for several years to extend the shelf-life of packaged foods [2,3]. The aim of this study was to investigate the effect of the modified atmosphere packaging of Spanish-style green olives in multi-layered pouches on the microbiological, physicochemical and sensory characteristics of cvs. Halkidiki and Conservolea olives. 


\section{Materials and Methods}

\subsection{Olive Samples and Packaging Conditions}

Samples of cv. Halkidiki and Conservolea pitted green olives processed by the Spanish method were packaged in high barrier multi-layered pouches $(113 \mu \mathrm{m}$ thickness; $\mathrm{O}_{2}$ permeability $\left.<1.5 \mathrm{~mL} / \mathrm{m}^{2} / 24 \mathrm{~h}\right)$ under modified atmospheres $\left(30 \% \mathrm{CO}_{2} / 70 \% \mathrm{~N}_{2}\right)$ and stored at room temperature for 12 months. Sampling was repeated every 30 days for 12 months.

\subsection{Microbiological Analyses}

Microbiological analyses were undertaken in olive samples every 30 days to determine the population dynamics of total viable counts (TVCs), lactic acid bacteria (LAB), yeasts and Enterobacteriaceae, as detailed elsewhere [4].

\subsection{Physicochemical and Sensory Analyses}

Additionally, $\mathrm{pH}$, titratable acidity, salinity, color and texture measurements were undertaken at the same time intervals as for microbiological analyses. The $\mathrm{pH}$ of the brine was measured using a digital $\mathrm{pH}$ meter. Acidity and salinity were determined by titration and expressed as a percentage $(w / w)$ of lactic acid and $(w / w)$ of $\mathrm{NaCl}$, respectively [5]. The color changes on the surface of olive drupes were measured using a Minolta type chromatometer (Nonastru, $3 \mathrm{nh}$, NS800) based on $L^{*}$ (lightness), $a^{*}$ (redness/greenness), and $b^{*}$ (yellowness/blueness) parameters [6]. Texture analysis was performed using a laboratory texturemeter [5-7]. The sensory characteristics of packaged olives were evaluated on a monthly basis by a taste panel according to the protocol of the International Olive Council [8,9].

\section{Results and Discussion}

\subsection{Microbiological Changes}

The evolution of the microbial consortia (TVC, LAB and yeasts) on olive drupes of cv. Halkidiki and Conservolea during storage under modified atmosphere is illustrated in Figure 1. Enterobacteriaceae could not be enumerated during storage in all samples. LAB were the dominant microbiota and ranged 5.4-6.4 log CFU/g and 4.5-6.2 log CFU/g for cv. Halkidiki and Conservolea, respectively. Moreover, yeasts were detected within the first 60 days of storage in lower populations compared to LAB, reaching maximum counts of $4.9 \log \mathrm{CFU} / \mathrm{g}$ and $5.4 \log \mathrm{CFU} / \mathrm{g}$ in cv. Halkidiki and Conservolea, respectively. However, no yeasts could be detected in olives after 90 days of storage in both varieties. The evolution of the microbial consortia observed in this study is in good agreement with previous researchers who confirmed the dominance of LAB and the co-existence of yeasts at lower populations $[4,10,11]$. 


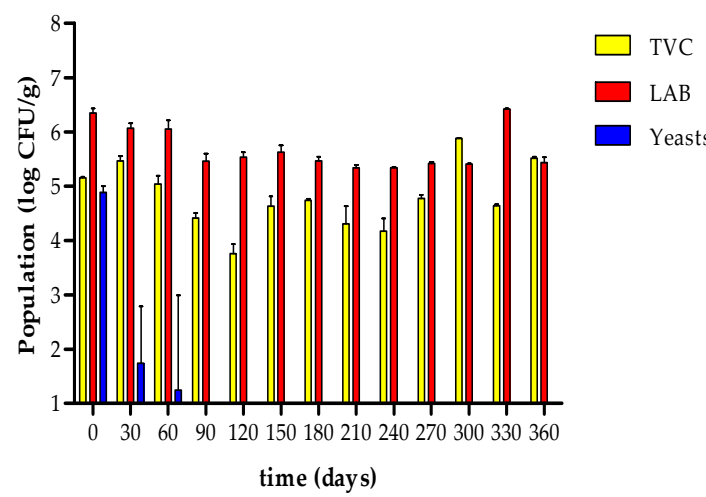

(a)

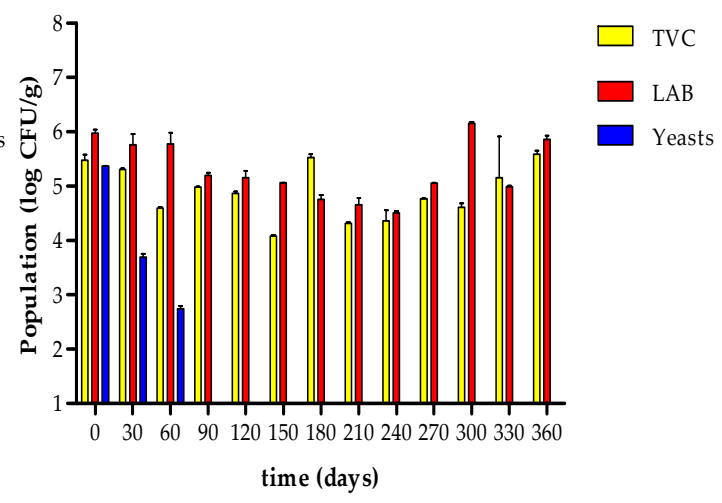

(b)

Figure 1. Changes in the population of total viable counts (TVCs), lactic acid bacteria (LAB), and yeasts on olive drupes of cv. Halkidiki (a) and Conservolea (b) during modified atmosphere packaging in multi-layered pouches. Data are average values of duplicate samples \pm standard deviation.

\subsection{Physicochemical Changes and Sensory Analyses}

During storage, the titratable acidity, $\mathrm{pH}$ and salt content of the packaged pitted olives were monitored for 12 months and the results are presented in Figure 2. The values of $\mathrm{pH}$ increased gradually from 3.51 to 4.19 , whereas the mean titratable acidity fluctuated between 0.2 and $0.4 \%(w / w$, lactic acid) in both cultivars. The salt content varied between $2.0-4.4 \%(w / w, \mathrm{NaCl})$ in all samples. The aforementioned changes in acidity, $\mathrm{pH}$ and salinity are in line with previous publications on packaged table olives of different cultivars $[4,6,11,12]$.

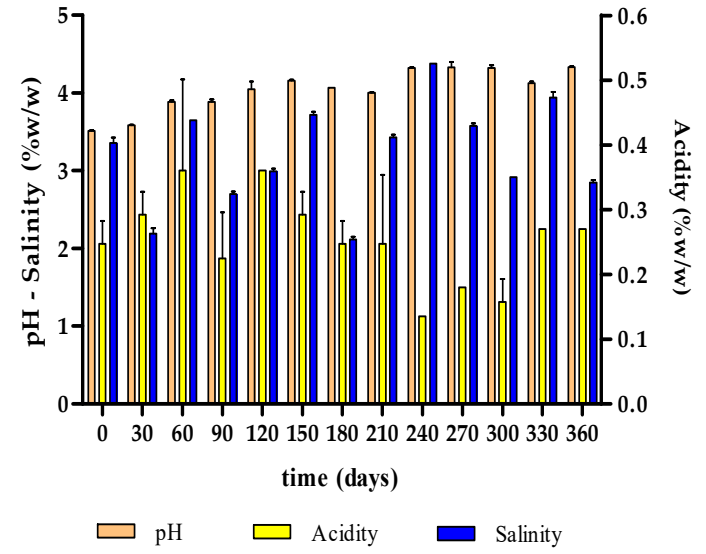

(a)

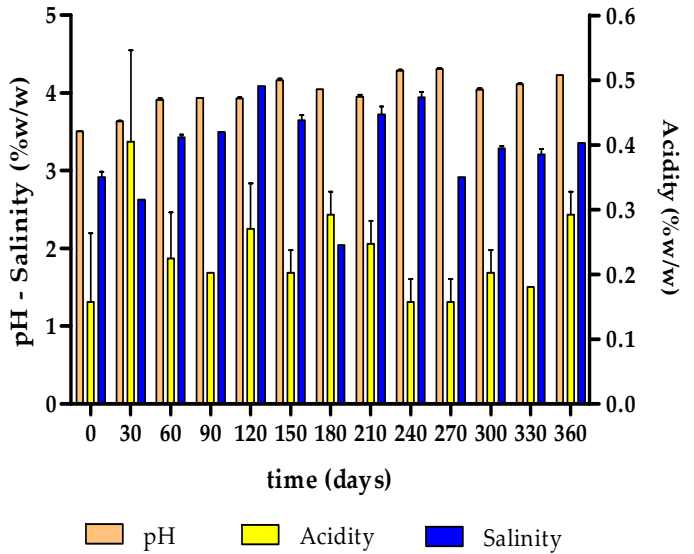

(b)

Figure 2. Changes in $\mathrm{pH}$, salt content and titratable acidity during the storage of packaged pitted green olives of $\mathrm{cv}$. Halkidiki (a) and Conservolea (b). Data are the average values of duplicate samples \pm standard deviation.

The color parameters $L^{*}, a^{*}$ and $b^{*}$ did not change during storage in both varieties. No statistically significant differences could be established for $L^{*}$ parameter corresponding to the brightness of olives. Additionally, the values of $a^{*}$ and $b^{*}$ parameters indicated the prevalence of green and yellow tonalities among samples, regardless of table olive variety [13]. The color of olives has a major impact on the green table olive market 
and consequently high brightness values and the prevalence of vivid green tonality are desirable characteristics [14].

The force needed to penetrate the olive drupe was considered as a measure of the olive's hardness. The observed values varied according to the table olive variety and storage time. Initially, the mean value of hardness was 15-20 N and after 360 days of storage, it decreased to $8 \mathrm{~N}$. According to previously published research, packaging in modified atmospheres or in aerobic conditions resulted in reduced firmness during storage [4]. Moreover, it was reported that vacuum-packed olives presented the lowest softening rate and hence the longest shelf-life followed by modified atmosphere and aerobic packaging [4].

During sensory assessment, the taste panel evaluated negative (e.g., abnormal fermentation), gustatory (salty, bitter and acid taste) and kinesthetic sensations (hardness, fibrousness, crunchiness). No perception of abnormal fermentation (zapateria, putrid, butyric) was noticed throughout storage. Furthermore, the majority of the sensory characteristics did not vary throughout storage in both varieties. According to the outcome of the sensory assessment, olive samples from cv. Halkidiki were bitter, acid, crisper and with increased fibrousness compared to cv. Conservolea. However, after 10 months of storage, all olive samples received approximately the same score with no important differences. Overall, all samples were characterized as "Extra" according to the protocol of sensory analysis of table olives established by the International Olive Council. The composition of the modified atmosphere is important since it affects the color and texture of olives, and consequently the judgement of the panelists [4].

\section{Conclusions}

The modified atmosphere packaging of Spanish-style green pitted olives in multi-layered pouches revealed stability with minor changes in terms of the microbiological and physicochemical characteristics of cv. Halkidiki and Conservolea olives during 12-month storage. From the microbiological perspective, LAB dominated the olive surface from the beginning of storage, whereas no yeasts could be detected after 3 months of storage. The dominance of $\mathrm{LAB}$ on the olives throughout the storage in populations exceeding $5.0 \log \mathrm{CFU} / \mathrm{g}$ combined the probiotic potential of this microbial group, creates a new perspective for the development of functional table olives. In addition, the absence of Enterobacteriaceae throughout packaging ensures the microbiological safety of the product. The changes in the physicochemical parameters were within acceptable limits to ensure the stability of the product during storage.

Author Contributions: Conceptualization, E.Z.P.; methodology, E.Z.P., G.-J.N. and A.T.; investigation, A.T.; resources, E.Z.P. and G.-J.N.; writing-original draft preparation, A.T.; writing-review and editing, E.Z.P.; visualization, A.T. and E.Z.P.; supervision, E.Z.P.; project administration, G.E.-P. and S.P.; funding acquisition, G.E.-P. and S.P. All authors have read and agreed to the published version of the manuscript.

Funding: This research has been co-financed by the European Union and Greek national funds through the Operational Program Competitiveness, Entrepreneurship and Innovation, under the call RESEARCH-CREATE-INNOVATE (project code: T1EDK-04110).

Institutional Review Board Statement: Not applicable

Informed Consent Statement: Not applicable

Data Availability Statement: Data available on request due to restrictions.

Conflicts of Interest: The authors declare no conflict of interest. 


\begin{tabular}{|c|c|}
\hline \multicolumn{2}{|c|}{ Abbreviations } \\
\hline LAB & Lactic acid bacteria \\
\hline TVC & Total viable counts \\
\hline MAP & Modified atmosphere packaging \\
\hline CFU & Colony Forming Units \\
\hline
\end{tabular}

\section{References}

1. Kazou, M.; Tzamourani, A.; Panagou, E.Z.; Tsakalidou, E. Unraveling the microbiota of natural black cv. Kalamata fermented olives through 16S and ITS metataxonomic analysis. Microorganisms 2020, 8, 672.

2. Değirmencioğlu, N.; Gürbüz, O.; Değirmencioğlu, A.; Şahan, Y.; Özbey, H. Effect of MAP and vacuum sealing on sensory qualities of dry-salted olives. Food Sci. Biotechnol. 2011, 20, 1307-1313.

3. Sánchez, A.H.; López-López, A.; Beato, V.M.; de Castro, A.; Montaño, A. Stability of color in Spanish-style green table olives pasteurized and stored in plastic containers. J. Sci. Food Agric. 2017, 97, 3631-3641.

4. Panagou, E.Z. Effect of different packing treatments on the microbiological and physicochemical characteristics of untreated green olives of the Conservolea cultivar. J. Sci. Food Agric. 2004, 84, 757-764.

5. Garrido-Fernández, A.; Fernández-Díez, M.J.; Adams, M.R. Table Olives: Production and Processing; Chapman \& Hall: London, UK, 1997.

6. Blana, V.A.; Polymeneas, N.; Tassou, C.C.; Panagou, E.Z. Survival of potential probiotic lactic acid bacteria on fermented green table olives during packaging in polyethylene pouches at 4 and $20^{\circ} \mathrm{C}$. Food Microbiol. 2016, 53, 71-75.

7. Fadda, C.; Del Caro, A.; Sanguinetti, A.M.; Piga, A. Texture and antioxidant evolution of naturally green table olives as affected by different sodium chloride brine concentrations. Grasas y Aceites 2014, 65, 1-10.

8. International Olive Council (IOC). Sensory Analysis of Table Olives. Available online: https://www.internationaloliveoil.org/wp-content/uploads/2019/11/COI-OT-MO.-1-Rev.2-2011-Eng.pdf (assessed on 18 October 2020).

9. Lanza, B.; Amoruso, F. Panel performance, discrimination power of descriptors, and sensory characterization of table olive samples. J. Sens. Stud. 2020, 35, 1-15.

10. Rodriguez-Gomez, F.; Romero-Gil, V.; Bautista-Gallego, J.; García-García, P.; Garrido-Fernández, A.; Arroyo-López, F.N. Production of potential probiotic Spanish-style green table olives at pilot plant scale using multifunctional starters. Food Microbiol. 2014, 44, 278-287.

11. Argyri, A.A.; Nisiotou, A.A.; Pramateftaki, P.; Doulgeraki, A.I.; Panagou, E.Z.; Tassou, C.C. Preservation of green table olives fermented with lactic acid bacteria with probiotic potential under modified atmosphere packaging. LWT Food Sci. Technol. 2015, $62,783-790$.

12. Doulgeraki, A.I.; Hondrodimou, O.; Iliopoulos, V.; Panagou, E.Z. Lactic acid bacteria and yeast heterogeneity during aerobic and modified atmosphere packaging storage of natural black Conservolea olives in polyethylene pouches. Food Control 2012, $26,49-57$.

13. Sánchez, A.H.; Romero, C.; Ramírez, E.; Brenes, M. Storage of mechanically harvested Manzanilla olives under controlled atmospheres. Postharvest Biol. Technol. 2013, 81, 60-65.

14. Rodriguez-Gomez, F.; Romero-Gil, V.; Arroyo-López, F.N.; Bautista Gallego, J.; García-García, P.; Garrido-Fernández, A. Effect of packaging and storage conditions on microbial survival, physicochemical characteristics and color of non-thermally preserved green Spanish-style Manzanilla olives. LWT Food Sci. Technol. 2015, 63, 367-375. 